\title{
Comparative analysis of gait and speech in Parkinson's disease: \\ hypokinetic or dysrhythmic disorders?
}

Stéphanie Cantiniaux ${ }^{1,2}$, Marianne Vaugoyeau ${ }^{2}$, Danièle Robert ${ }^{3}$, Christine Horrelou-Pitek ${ }^{3}$, Julien Mancini $^{4,5}$, Tatiana Witjas ${ }^{1}$, Jean-Philippe Azulay ${ }^{1,2}$

1. Department of Neurological Sciences, Movement Disorders Unit, hôpital de la Timone, 264, rue Saint-Pierre, 13385 Marseille cedex 05, France

2. CNRS- Université Provence, Site Saint Charles, DPA-UMR 6149, Pôle 3C, Case B, 3 Place Victor Hugo, 13331 Marseille Cedex 03, France

3. Department of Otorhinolaryngology, hôpital de la Timone, 264, rue Saint-Pierre, 13385

Marseille cedex 05, France

4. Aix Marseille Université, LERTIM, EA 3283, Marseille, F-13385 France;

5. Department of Public Health and Medical Information t, hôpital de la Timone, 264, rue Saint-Pierre, 13385 Marseille cedex 05, France

Corresponding author jean-Philippe Azulay, Service de Neurologie et Pathologie du Mouvement. Hôpital de la Timone, 264, rue Saint-Pierre, 13385 Marseille cedex 05, France and Jean-Philippe Azulay,

e-mail : jean-philippe.azulay@ap-hm.fr

Key Words: Parkinson's disease, Locomotion, Speech, Deep Brain Stimulation

Word count : 3578

Abstract : 260 


\section{Abstract:}

Gait and speech are automatic motor activities which are frequently impaired in Parkinson's disease (PD). Obvious clinical similarities exist between these disorders but were never investigated. We propose to determine whether there exist any common features in PD between spatiotemporal gait disorders and temporal speech disorders.

Gait and speech were analyzed on eleven PP undergoing deep-brain-stimulation of the subthalamic-nucleus (STN-DBS) and eleven control subjects (CS) under 3 conditions of velocity (natural, slow and speed). The patients were tested with and without L-Dopa and stimulator ON or OFF. Locomotor parameters were recorded using an optoelectronic system. Speech parameters were recorded with a headphone while subjects were reading a short paragraph.

The results confirmed that PP walk and read more slowly than controls. Patient's difficulties in modulating walking and speech velocities seem to be due mainly to an inability to internally control the step length and the interpause-speech duration ISD.

STN-DBS and levodopa increased patients' walking velocity by increasing the step length. STN-DBS and levodopa had no effect on speech velocity but restored the patients' ability to modulate the ISD. The walking cadence and speech index of rythmicity (SPIR) tended to be lower in patients and were not significantly improved by STN-DBS or levodopa. Speech and walking velocity as well as ISD and step length were correlated in both groups. Negative correlations between SPIR and walking cadence were observed in both groups

Similar fundamental hypokinetic impairment and probably a similar rhythmic factor affected similarly the patients' speech and gait. These results suggest a similar physiopathological process in both walking and speaking dysfunction. 


\section{Introduction}

Basal ganglia dysfunction leads to the lack of automatic execution of learned motor sequences (Marsden, 1982) and to the development of Parkinsonian motor symptoms (Lee et al., 1994), including changes in gait and speech.

Gait disorders in PD (Hoehn and Yahr, 1967), constitute one of the main factors affecting patients' independence and quality of life (Koplas et al., 1999). PP walk slower, with shorter step length and a compensatory increased walking cadence (Blin et al, 1990, Morris et al., 1994a, b). Levodopa improves spatiotemporal parameters such as walking velocity and step length, but not temporal parameters such as step time, swing time and walking cadence (Azulay et al., 1996; Blin et al., 1990; Pedersen et al., 1991). Studies have shown that Sub-Thalamic-Nucleus Deep Brain Stimulation (STN-DBS) improves walking velocity by increasing the step length without affecting the walking cadence (Faist et al., 2001; Lubik et al., 2006) while a modulation of cadence has been reported by others (Stolze et al., 2001).

Approximately $70 \%$ of PP experience speech difficulties attributed to bradykinetic and hypokinetic articulatory movements, orofacial hypomimy, rigidity and rest tremor (Hartelius and Svensson, 1994).

PPs' speech timing deficits seem to have some points in common with their spatiotemporal gait deficits. Speech velocity often decreases in PP (Canter, 1963; Metter and Hanson, 1986) just like walking velocity. The interpauses speech duration (ISD) is usually shortened in PP (Forrest et al., 1989; Hammen and Yorkston, 1996), in line with the step length.

PP are subject to dysprosody, and show less variability in the fundamental frequency and intensity and more speech velocity and pausing abnormalities than control subjects (CS) (Canter, 1963; Harel et al., 2004; Kegl et al., 1999) . 
Dysfluent behavior has also been described in PP (Benke et al., 2000), consisting mainly of repetitive speech phenomena involving the repetition of syllables, words or phrases. Skodda and Schlegel (2008) reported that during a standardized reading task, the speech velocity of PP accelerates more strongly while speaking and the total number of pauses decreases significantly, which indicates that their speech rhythm and timing organization processes are impaired. Physicians often have the feeling that paroxystic speech velocity accelerations develop in line with gait festinations and dysrhythmic pauses and that repetitive speech phenomenon such as stuttering, iterations and palilalia are somewhat comparable to freezing of gait. Few studies have focused on the effects of levodopa on speech timing parameters. Levodopa does not improve speech velocity (Wolfe et al., 1975) or stuttering (Anderson et al., 1999; Benke et al., 2000). Speech dysfluencies may be due to an increase or a decrease in the dopamine levels present in the brain Two studies have shown that selective left-side STN-DBS may have negative effects on prosody (Santens et al., 2003; Wang et al., 2003), but the quantitative effects of STN-DBS on specific temporal speech parameters have never been assessed.

Our aim was to compare gait and speech patterns of PP and CS, and to analyse the patients' responses to levodopa and STN-DBS. Another aim was to determine whether there exist any correlations between the spatiotemporal gait and temporal speech parameters with a view to establishing whether, in line with what occurs with gait, hypokinesia may be mainly responsible for these patients' speech timing disorders and whether accelerated timing strategies may be used as compensatory mechanisms.

\section{MATERIAL AND METHODS}

\section{Subjects}


Eleven PP, 7 males and 4 females (mean age 64.5) and 11 age-matched CS, 7 males and 4 females (mean age 66.3) with no neurological history took part in these experiments after giving their informed consent. The project was approved by the local ethical committee. All the PP had undergone bilateral STN-DBS and were stable when the study was conducted. PP and controls with any other disorder possibly affecting their walking or speaking abilities were excluded from the study.

PPs' level of functional disability was determined on the Unified Parkinson's Disease Rating Scale (UPDRS) motor and axial scores in four treatment conditions (OFF and ON states of STN-DBS and levodopa). The patients' characteristics are given in Table 1.

\section{Test procedure}

Gait and speech recordings were conducted in the four treatment conditions, always in the same order. Gait recordings were run always just before speech recordings.

1. PP were tested in the OFF STN-DBS state at least 12 hours after undergoing STNDBS treatment and in the OFF levodopa state at least 12 hours after their last dose of dopaminergic treatment (DT) $(\mathrm{OFF} / \mathrm{OFF})$

2. the PP were tested in the ON STN-DBS state at least 1 hour after undergoing STNDBS treatment and in the ON levodopa state at the peak effect of a suprathreshold dose of levodopa (ON/ON).

3. the PP were tested in the ON STN-DBS state at least 12 hours after undergoing STNDBS treatment and in the OFF levodopa state at least 12 hours after taking their last DT dose (ON/OFF). 
4. the PP were tested in the OFF STN-DBS state at least 3 hours after undergoing STNDBS treatment and in the ON levodopa state at the peak effect of a suprathreshold dose of levodopa (OFF/ON).

The gait and speech recordings were conducted just once on the CS.

3. Gait recordings

3.1 Tasks

Subjects were instructed to walk on level ground at their natural speed (NS), slowest as compared with the first task (SS) and as fast as possible without running (FS).

\subsection{Experimental recordings}

Kinematic analysis was performed with the ELITE TV image processing system (Ferrigno and Pedotti, 1985). 22 reflective markers $1 \mathrm{~cm}$ in diameter were taped onto the subjects' skin at specific symmetrical anatomical points. The recordings were taken with 6 TV cameras at a sampling rate of $100 \mathrm{~Hz}$.

\subsection{Gait parameters}

Based on the kinematic recording, temporal and spatial gait parameters were measured during each trial: walking velocity $(\mathrm{m} / \mathrm{s})$, step length $(\mathrm{mm})$ and walking cadence (step/min).

4. Speech recordings

4.1 Tasks

Subjects had to read a short printed text at their natural speed (NS), slowest as compared with the natural condition (SS) and as fast as possible (FS).

\subsection{Experimental recordings}


Subjects were seated in a quiet room. Temporal acoustic recordings obtained via a headphone were analyzed using the Prosody ${ }^{\mathrm{R}}$ software program, which is part of the Software Environment for Speech Analysis and Evaluation $\left(\mathrm{SESANE}^{\mathrm{R}}\right)$.

\subsection{Speech parameters}

Based on the acoustic data the following parameters were measured at each trial:

- Speech velocity (syllables/second)

- Interpause speech duration (ISD in seconds): It corresponds to the time interval between two pauses. It was determined by tallying the duration occurring between two pauses.

- Speech Index of Rhythmicity (SPIR): this is an index to evaluate speech rhythmicity in the same way as the walking cadence. As the alternation between pauses and speech gives rhythm to speech just as the alternation between steps and double foot support phases gives rhythm to gait, SPIR corresponds the number of speech interpauses per minute.

\section{Statistical procedure}

Because of the small size and the non normal distribution of our samples, all the tests applied were non parametric tests. For each subject and condition, all variables have been averaged over the three trials. In the OFF/OFF situation, two PP were unable to walk. Nevertheless, we have used the data of these patients in the other conditions of treatment. The statistics given are medians and interquartiles.

Differences between CS and PP were tested with a Mann-Whitney $U$ test. The task effect in each group and the treatment effect in the PP group were analyzed with a Friedman ANOVA. In order to isolate statistical differences when the hypothesis of differences was verified we have realised a multiple comparison procedures (post-hoc tests) using a Student- 
Newman-Keuls (SNK) test. The correlations between gait and speech parameters were tested with a Spearman test.

\section{RESULTS}

1. Gait analysis

\subsection{Effects of PD}

In order to assess the effects of PD on gait parameters, the gait characteristics of $\mathrm{PP}$ in the OFF/OFF state of treatment were compared with those of the CS.

Whatever experimental situations, gait velocity of PP was slower than that of CS (T $(1.18)=61, \mathrm{p}<0.05 ; \mathrm{T}(1.18)=62, \mathrm{p}<0.05$ and $\mathrm{T}(1.18)=58, \mathrm{p}<0.05$ in conditions NS, SS and FS, respectively); the mean step length was shorter $(\mathrm{T}(1.18)=57, \mathrm{p}<0.01 ; \mathrm{T}(1.18)=59, \mathrm{p}<0.01$ and $\mathrm{T}(1.18)=59, \mathrm{p}<0.01$ in conditions NS, SS and FS, respectively); whatever the experimental task the cadence was similar in both groups.

\subsection{Effects of treatment}

The gait characteristics of PP in each mode of treatment are given in figure 1.

\section{a. Walking Velocity}

The statistical analysis revealed a global effect of the treatment on walking velocity in all experimental condition $\left(\mathrm{chi}^{2}=9.41, \mathrm{p}<0.01\right.$; $\mathrm{chi}^{2}=17.93, \mathrm{p}<0.001$ and $\mathrm{chi}^{2}=15, \mathrm{p}<0.01$ for NS, SS, and FS respectively). Walking velocity increased similarly with levodopa, DBS and combination of both compared with the complete OFF state.

\section{b. Step Length}


In the NS condition, we showed a significant global effect of treatment on step length $\left(\mathrm{chi}^{2}=15.27, \mathrm{p}=0.002\right)$. Statistical analysis showed a significant increase of the step length in the ON/ON state of treatment in comparison with the other states. The SNK post hoc analysis revealed a significant decrease of the step length in the OFF/OFF state of treatment as compared with the ON/OFF and OFF/ON states of treatment. No significant differences were observed between OFF/ON and ON/OFF states, indicating that levodopa and DBS similarly improved step length and that the combination of both therapies had an additional effect.

In SS and FS, we showed a significant global effect of treatment on step length $\left(\mathrm{chi}^{2}=15.8, \mathrm{p}=0.001\right.$ and $\mathrm{chi}^{2}=11.8, \mathrm{p}<0.01$, for SS and FS respectively). The step length was significantly shorter in the OFF/OFF state than in the other. No significant differences were observed between the states OFF/ON, ON/OFF or ON/ON.

c. Walking cadence

In the NS and SS conditions, the walking cadence did not differ significantly between all states of treatment. In the SS condition, a significant overall effect of treatment was observed $\left(\mathrm{chi}^{2}=8.33, \mathrm{p}<0.05\right)$. The multiple comparisons revealed that the walking cadence was significantly lower in the OFF/OFF state of treatment than in the other.

\section{INSERT FIGURE 1 ABOUT HERE}

\subsection{Task effect}

a. Walking velocity

A significant effect of task was observed in $\mathrm{CS}\left(\mathrm{chi}^{2}=22, \mathrm{p}<0.001\right)$ and in treated PP $\left(\mathrm{chi}^{2}=22, \mathrm{p}<0.001 ; \mathrm{chi}^{2}=20, \mathrm{p}<0.001\right.$ and $\mathrm{chi}^{2}=18.2, \mathrm{p}<0.001$ in the 
OFF/ON, ON/OFF and ON/ON states, respectively): The walking velocity increased significantly between SS and FS. Without treatment a significant effect of task was also observed in PP $\left(\mathrm{chi}^{2}=12.67, \mathrm{p}<0.001\right)$ but interestingly, no significant difference was found between NS and FS, indicating that PP were unable to increase significantly their velocity.

b. Step length

A significant effect of task on step length was observed in $\mathrm{CS}\left(\mathrm{chi}^{2}=22, \mathrm{p}<0.001\right)$ and in treated PP, whatever the treatment the step length increased significantly between the $\mathrm{SS}$ and the FS condition $\left(\mathrm{chi}^{2}=22, \mathrm{p}<0.001 ; \mathrm{chi}^{2}=16.8, \mathrm{p}<0.001\right.$ and $\mathrm{chi}^{2}=14.6$, $\mathrm{p}<0.001$ in the $\mathrm{OFF} / \mathrm{ON}, \mathrm{ON} / \mathrm{OFF}$ and $\mathrm{ON} / \mathrm{ON}$ states, respectively). In untreated $\mathrm{PP}$ a significant effect of task was also observed $\left(\mathrm{chi}^{2}=11.56, \mathrm{p}=0.001\right)$, but no significant difference was found to exist between NS and FS conditions.

c. Walking cadence

A significant effect of task was observed in CS $\left(\mathrm{chi}^{2}=16.55, \mathrm{p}<0.001\right)$ and in treated PP $\left(\mathrm{chi}^{2}=16.22, \mathrm{p}<0.001 ; \mathrm{chi}^{2}=22, \mathrm{p}<0.001 ; \mathrm{chi}^{2}=18.2, \mathrm{p}<0.001\right.$ and $\mathrm{chi}^{2}=20, \mathrm{p}<0.001$ for $\mathrm{OFF} / \mathrm{OFF}, \mathrm{OFF} / \mathrm{ON}, \mathrm{ON} / \mathrm{OFF}$ and $\mathrm{ON} / \mathrm{ON}$ states of treatment, respectively). The walking cadence increased significantly between SS, NS and FS.

\section{INSERT FIGURE 2 ABOUT HERE}

2. Speech analysis

2.1 Effects of Parkinson's Disease 
During NS and SS tasks, speech velocity did not differ significantly between the two groups. In the FS task the speech velocity was significantly lower in $\mathrm{PP}(\mathrm{T}=93.5, \mathrm{p}<0.05)$.

In the NS task, ISDs' values were similar in both groups. In the SS task, ISD's value of PP was significantly longer than that of CS $(\mathrm{T}=160.5, \mathrm{p}<0.05)$; whereas in the FS task, the ISD of PP was significantly shorter than that of CS $(\mathrm{T}=91, \mathrm{p}<0.05)$.

In NS and FS tasks, the SPIR did not differ significantly between the two groups. In the SS task, the SPIR values of PP were significantly lower than those of CS $(T=93, p<0.05)$.

\subsection{Effects of treatment}

In order to assess the effects of treatment, speech performances of PP were compared depending on the treatment undergone. The speech performances of PP are given in figure 3 .

a. Speech velocity

Whatever the experimental condition, no significant differences were observed between the four states of treatment.

b. ISD

Concerning the ISD, the Friedman's ANOVA showed significant differences between the four states of treatment during NS $\left(\mathrm{chi}^{2}=9.275, \mathrm{p}<0.05\right)$ and FS condition $\left(\mathrm{chi}^{2}=1.175\right.$, $\mathrm{p}<0.01)$ but not during SS condition. ISD increased significantly with levodopa, DBS and combination of both therapies as compared with the complete OFF state.

c. SPIR 
The SPIR did not differ significantly between any of the states of treatment during NS and SS conditions. During FS condition, the statistical analysis showed the existence of a significant overall effect of treatment on SPIR $\left(\mathrm{chi}^{2}=8.46, \mathrm{p}<0.05\right)$ which were significantly higher in the OFF/OFF state of treatment than in the other states.

\section{INSERT FIGURE 3 ABOUT HERE}

\subsection{Task effects}

a. Speech velocity

A significant effect of task on speech velocity was observed in CS $\left(\mathrm{chi}^{2}=16.545\right.$, $\mathrm{p}<0.01)$ and in treated PP $\left(\mathrm{chi}^{2}=14.727, \mathrm{p}<0.01 ; \mathrm{chi}^{2}=20.182, \mathrm{p}<0.001\right.$ and $\mathrm{chi}^{2}=14.364, \mathrm{p}<0.01$ in the $\mathrm{OFF} / \mathrm{ON}, \mathrm{ON} / \mathrm{OFF}$ and $\mathrm{ON} / \mathrm{ON}$ states, respectively): the speech velocity increased significantly between the SS and the FS condition. A significant effect of task was also observed in the OFF/OFF PP $\left(\operatorname{chi}^{2}=17.636, p<0.01\right)$ : speech velocity increased significantly between NS and FS condition, but not between SS and NS conditions.

b. ISD

A significant effect of task on ISD was found in CS $\left(\mathrm{chi}^{2}=13.273, \mathrm{p}<0.01\right)$ and in treated PP $\left(\mathrm{chi}^{2}=10.093, \mathrm{p}<0.01 ; \mathrm{chi}^{2}=8.727, \mathrm{p}<0.05\right.$ and $\mathrm{chi}^{2}=13.273, \mathrm{p}<0.01$ in the $\mathrm{OFF} / \mathrm{ON}, \mathrm{ON} / \mathrm{OFF}$ and $\mathrm{ON} / \mathrm{ON}$ states, respectively): the ISD increased significantly between the SS and FS condition. It is worth noting that the increase of the ISD between NS and FS was only significant in the ON/ON state of treatment. In PP in the OFF/OFF state of treatment, no significant differences were detected. 


\section{c. SPIR}

A significant effect of task on SPIR was observed in CS $\left(\mathrm{chi}^{2}=8.909, \mathrm{p}<0.001\right)$ : the SPIR values decreased significantly in this group between SS and NS and between NS and FS. No significant effects of task on SPIR were detected in PP.

\section{INSERT FIGURE 4 ABOUT HERE}

\section{Correlations between gait and speech parameters}

In order to measure the strength of association between gait and speech parameters we have used the Spearman's coefficient of correlation. We have correlated gait velocity with speech velocity, walking cadence with SPIR and step length with ISD. We have brought together all the velocity of tasks executions.

\subsection{Statistical correlations between walking velocity and speech velocity}

Significant correlations were found to exist between walking and speech velocities in CS $(r=0.84, p<0.001)$, in PP in the OFF/OFF $(r=0.717, p<0.001)$, in the ON/OFF $(r=0.505$, $\mathrm{p}<0.01)$, and in the OFF/ON states of treatment $(\mathrm{r}=0.4325, \mathrm{p}<0.05)$ but not in the ON/ON sate.

\subsection{Correlations between step length and ISD}

A significant correlation was found to exist between step length and ISD in CS $(\mathrm{r}=0.609, \mathrm{p}<0.001)$, in PP in the ON/OFF $(\mathrm{r}=0.486, \mathrm{p}<0.01)$ and ON/ON $(\mathrm{r}=0.421, \mathrm{p}<0.05)$ state of treatment. No statistical correlations were observed in the OFF/OFF and OFF/ON states of treatment.

3.3. Correlations between walking cadence and SPIR 
A significant negative correlation was found between walking cadence and SPIR in $\mathrm{CS}(\mathrm{r}=-0.702, \mathrm{p}<0.001)$ in PP in the OFF/OFF $(\mathrm{r}=-0.418, \mathrm{p}<0.05)$ and in the OFF/ON state of treatment $(\mathrm{r}=-0.438, \mathrm{p}<0.05)$, but not in PP in the ON/OFF or ON/ON states of treatment.

\section{DISCUSSION:}

Our results demonstrate large similarities between the gait's pattern and speech's involvement in PD and in their responses to STN-DBS and levodopa treatment

\section{Gait and speech impairments in PD}

The spatiotemporal gait disorders observed in PD are in line with those previously described: without treatment, walking velocity and step length of PP are decreased while walking cadence is not significantly different (Azulay et al., 1996; Morris et al., 1994b; Sofuwa et al., 2005).Neither walking velocity nor step length increased when the untreated patients were asked to walk as fast as possible, suggesting that they were unable to regulate their step length on the basis of internal control mechanisms. Oppositely, the patients' internal walking cadence regulation process seems to be preserved, since their walking cadence increased significantly between SS and NS and between NS and FS. This fundamental hypokinetic deficit has previously been described by Morris et al. (1994b).

The results of the speech analysis brought to light the existence of speech timing disorders showing some analogies with the gait impairments. Without any treatment, PP tended to speak more slowly than the CS, their ISD often tended to decrease in comparison with the control values, in parallel with the decreasing step length. The SPIR showed some similarities with the walking cadence, since it was equal to the control values at NS and FS. 
Few data are available to compare our results, Canter (1963) and Metter and Hanson (1986) observed changing speech velocities in PP. The ISD has often been reported to be shorter in PP than in CS during both reading and spontaneous speech (Forrest et al., 1989; Hammen and Yorkston, 1996). The speech velocity also changes with the co-existence of bradylalia and tachylalia (Gentil et al., 1995). The present results show in addition that the speech velocity of PP without treatment did not differ between SS and NS, and that their ISD remained unchanged under all the experimental conditions. In line with the changes observed in their walking patterns, PP therefore have some difficulty in modulating their speech velocities because they are no longer able to internally control the ISD. However, the internal speech rhythm regulation processes seem to be also impaired, since the SPIR remained unchanged at NS and FS condition.

\section{Effect of levodopa and STN-DBS on gait and speech deficits}

As regards the patients' responses to treatment, their walking velocities were greatly improved by both STN-DBS and levodopa, probably due to an increase in the step length, whereas the walking cadence remained unchanged in NS and FS. In all tasks, no significant difference between STN-DBS and levodopa was observed, but a cumulative effect was observed in the NS condition. Two previous studies show that STN-DBS, like levodopa, improves walking velocity by increasing step length without changing walking cadence (Faist et al., 2001; Lubik et al., 2006). These findings suggest that STN-DBS may involve the same action mechanisms as levodopa via the dopaminergic basal ganglia loop while the cadence, which is a rhythmic parameter, is probably regulated by a non dopaminergic structure.

Levodopa and STN-DBS had no effect on the patients' speech velocity but increased the ISD. Combined treatment restored the patients' ability to modulate their ISD. The ISD 
therefore seems to be the main clinical manifestation of speech akinesia, since this parameter is regulated by the dopaminergic basal ganglia loop. The effects of treatment on the SPIR were often not significant. This finding probably means that in the case of both speech and gait, the rhythm is regulated by a non dopaminergic structure.

\section{Gait and speech deficits in PD: a common feature?}

This is the first time that correlations have been made between gait and speech parameters in PD. In CS and PP undergoing no treatment or under STN-DBS treatment alone, a significant correlation was found between walking velocity and speech velocity. In other words, CS and PP who walk faster also speak faster. This outcome seems to be modified by levodopa.

A significant positive correlation between step length and ISD was also found. This confirms that step length and ISD decrease are clinical manifestations of both gait and speech akinesia, that they are both responsive to levodopa and STN-DBS and that just as akinesia is the main gait deficit, speech akinesia may be the main speech deficit in PD. The negative correlation between walking cadence and SPIR in CS underlines the different strategies used to modulated gait and speech velocity: to walk faster, CS increase the number of steps they make, whereas to speak faster, they decrease the number of speech interpauses and pauses. This result suggests that the SPIR is a good index to analyse speech rhythmicity, just as the walking cadence is a good index to analyse gait rhythmicity. In PD, a similar phenomenon was observed only under levodopa suggesting that walking cadence and SPIR are regulated by different non dopaminergic structures and that rhythmic perturbations do not always affect

speech and gait concurrently, but that they may occur earlier in speech, where a two-fold 
deficit occurs due to the patients' inability to internally regulate either the number of pauses or the ISD.

Conclusions: As recently suggested by Moreau et al. (2007), who established that oral festinations were correlated with gait festinations in PP, the present results underline several common features between gait and speech troubles in PD. The decrease in PPs' walking velocity and speech velocity result from a decrease in step length and ISD: these akinetic parameters, which are strongly correlated, are regulated by the dopaminergic basal ganglia loop. Walking cadence and SPIR, which are the rhythmic gait and speech parameters, do not seem to be regulated by a non dopaminergic structure.

Experimental studies on animals have shown that the PPN of the MLR (Aziz et al., 1998) is involved in the regulation of the locomotor rhythm (Garcia-Rill and Skinner, 1988). On the basis of our results, we propose an equivalent model for the regulation of the spatial and rhythmic parameters of gait and the temporal and rhythmic parameters of speech (figure 5). Further studies are required to confirm such hypothesis and determine what structures are involved.

\section{INSERT FIGURE 5 ABOUT HERE}

Acknowledgements: We are grateful to all the subjects who participated in this study and to Dr Jessica Anderson-Blanc for revising the English manuscript. 


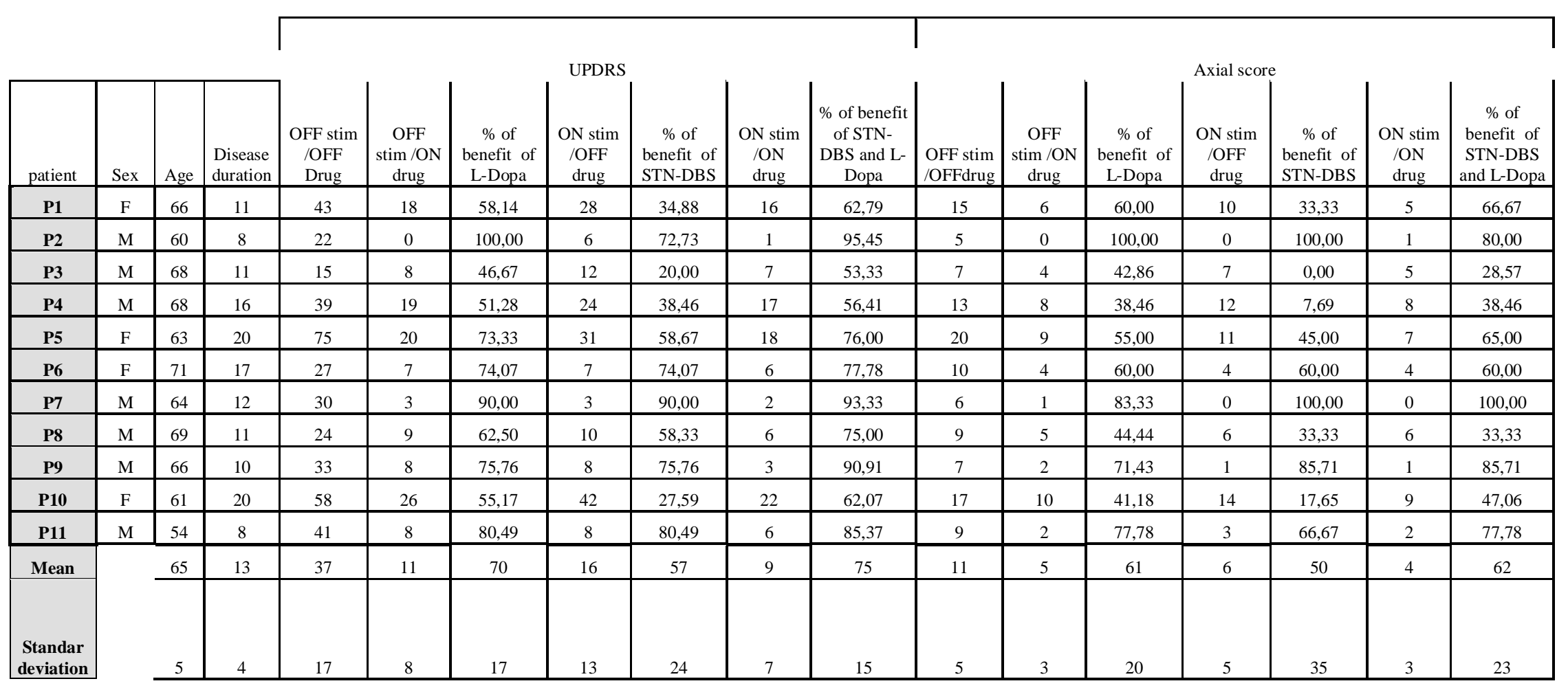

Table 1 


\section{FIGURE CAPTIONS:}

TABLE 1: Medical characteristics of the 11 Parkinsonian patients: sex, age and disease duration, UPDRS part III and axial scores, effect of L-Dopa and STN in \% iof the OFF/OFF score, in 4 states of treatment performed at the time of testing. The axial score was the sum of items numbers 18, 19, 22, 27, 28, 29 and 30 in the motor UPDRS score.

FIGURE 1: Task effect on gait parameters: From top to the bottom, medians and quartiles of walking velocity, step length and walking cadence values recorded in Parkinsonian patients in the 3 experimental conditions (from the left to the right) natural speed, slow speed and fast speed) in the 4 states of treatment, from left to right: without treatment (OFF/OFF), without STN-DBS but with levodopa (OFF/ON), with STN-DBS but without levodopa (ON/OFF) and with both STN-DBS and levodopa (ON/ON).

FIGURE 2: Treatment effect on gait parameters: Medians and quartiles of walking velocity, step length and walking cadence values recorded in the natural speed (in white), in the slow speed (in grey) and in the fast speed (in black) conditions, from left to right: without treatment (OFF/OFF), without STN-DBS but with levodopa (OFF/ON), with STN-DBS but without levodopa (ON/OFF) and with both STN-DBS and levodopa (ON/ON) and in control subjects (CS).

FIGURE 3: Task effects on speech parameters : Medians and quartiles of speech velocity, ISD and speech index of rythmicity (SPIR) recorded in Parkinsonian patients in the 3 
experimental conditions (natural speed, slow speed and fast speed) in the 4 states of treatment, from left to right: without treatment (OFF/OFF), without STN-DBS but with levodopa (OFF/ON), with STN-DBS but without levodopa (ON/OFF) and with both STN-DBS and levodopa $(\mathrm{ON} / \mathrm{ON})$.

FIGURE 4: Treatment effects on speech parameters : Medians and quartiles of speech velocity, ISD and speech index of rythmicity (SPIR) recorded in the natural speed (in white), in the slow speed (in grey) and in the fast speed (in black) conditions, from left to right: without treatment (OFF/OFF), without STN-DBS but with levodopa (OFF/ON), with STNDBS but without levodopa (ON/OFF) and with both STN-DBS and levodopa (ON/ON) and in control subjects (CS).

FIGURE 5: Model for the regulation of the spatial and rhythmic parameters of gait (left) and the temporal and rhythmic parameters of speech (right). 


\section{REFERENCES}

1. Anderson JM, Hughes JD, Rothi LJ, Crucian GP, Heilman KM. Developmental stuttering and Parkinson's disease: the effects of levodopa treatment. J Neurol Neurosurg Psychiatry 1999; 66: 776-8.

2. Aziz TZ, Davies L, Stein J, France S. The role of descending basal ganglia connections to the brain stem in parkinsonian akinesia. Br J Neurosurg 1998; 12: 245-9.

3. Azulay JP, Van Den Brand C, Mestre D, Blin O, Sangla I, Pouget J, et al. [Automatic motion analysis of gait in patients with Parkinson disease: effects of levodopa and visual stimulations]. Rev Neurol (Paris) 1996; 152: 128-34.

4. Benke T, Hohenstein C, Poewe W, Butterworth B. Repetitive speech phenomena in Parkinson's disease. J Neurol Neurosurg Psychiatry 2000; 69: 319-24.

5. Blin O, Ferrandez AM, Pailhous J, Serratrice G. [A new method of quantitative analysis of parkinsonian gait: report of 6 patients]. Rev Neurol (Paris) 1990; 146: 48-50.

6. Canter GJ. Speech Characteristics of Patients with Parkinson's Disease: I. Intensity, Pitch, and Duration. J Speech Hear Disord 1963; 28: 221-9.

7. Faist M, Xie J, Kurz D, Berger W, Maurer C, Pollak P, et al. Effect of bilateral subthalamic nucleus stimulation on gait in Parkinson's disease. Brain 2001; 124: 1590-600.

8. Ferrigno G, Pedotti A. ELITE: a digital dedicated hardware system for movement analysis via real-time TV signal processing. IEEE Trans Biomed Eng 1985; 32: 943-50.

9. Forrest K, Weismer G, Turner GS. Kinematic, acoustic, and perceptual analyses of connected speech produced by parkinsonian and normal geriatric adults. J Acoust Soc Am 1989; 85: 2608-22.

10. Garcia-Rill E, Skinner RD. Modulation of rhythmic function in the posterior midbrain. Neuroscience 1988; 27: 639-54.

11. Gentil M, Pollak P, Perret J. [Parkinsonian dysarthria]. Rev Neurol (Paris) 1995; 151: 105-12.

12. Goberman AM, Blomgren M. Parkinsonian speech disfluencies: effects of L-dopa-related fluctuations. J Fluency Disord 2003; 28: 55-70. 
13. Hammen VL, Yorkston KM. Speech and pause characteristics following speech rate reduction in hypokinetic dysarthria. J Commun Disord 1996; 29: 429-44; quiz 444-5.

14. Harel B, Cannizzaro M, Snyder PJ. Variability in fundamental frequency during speech in prodromal and incipient Parkinson's disease: a longitudinal case study. Brain Cogn 2004; 56: 24-9.

15. Hartelius L, Svensson P. Speech and swallowing symptoms associated with Parkinson's disease and multiple sclerosis: a survey. Folia Phoniatr Logop 1994; 46: 9-17.

16. Hoehn MM, Yahr MD. Parkinsonism: onset, progression and mortality. Neurology 1967; 17: 427-42.

17. Kegl J, Cohen H, Poizner H. Articulatory consequences of Parkinson's disease: perspectives from two modalities. Brain Cogn 1999; 40: 355-86.

18. Koplas PA, Gans HB, Wisely MP, Kuchibhatla M, Cutson TM, Gold DT, et al. Quality of life and Parkinson's disease. J Gerontol A Biol Sci Med Sci 1999; 54: M197-202.

19. Lee CS, Schulzer M, Mak EK, Snow BJ, Tsui JK, Calne S, et al. Clinical observations on the rate of progression of idiopathic parkinsonism. Brain 1994; 117 ( Pt 3): 501-7.

20. Lubik S, Fogel W, Tronnier V, Krause M, Konig J, Jost WH. Gait analysis in patients with advanced Parkinson disease: different or additive effects on gait induced by levodopa and chronic STN stimulation. J Neural Transm 2006; 113: 163-73.

21. Marsden CD. The mysterious motor function of the basal ganglia: the Robert Wartenberg Lecture. Neurology 1982; 32: 514-39.

22. Metter EJ, Hanson WR. Clinical and acoustical variability in hypokinetic dysarthria. J Commun Disord 1986; 19: 347-66.

23. Moreau C, Ozsancak C, Blatt JL, Derambure P, Destee A, Defebvre L. Oral festination in Parkinson's disease: biomechanical analysis and correlation with festination and freezing of gait. Mov Disord 2007; 22: 1503-6.

24. Morris ME, Iansek R, Matyas TA, Summers JJ. Ability to modulate walking cadence remains intact in Parkinson's disease. J Neurol Neurosurg Psychiatry 1994a; 57: 1532-4.

25. Morris ME, Iansek R, Matyas TA, Summers JJ. The pathogenesis of gait hypokinesia in Parkinson's disease. Brain 1994b; 117 ( Pt 5): 1169-81. 
26. Pedersen SW, Eriksson T, Oberg B. Effects of withdrawal of antiparkinson medication on gait and clinical score in the Parkinson patient. Acta Neurol Scand 1991; 84: 7-13.

27. Santens P, De Letter M, Van Borsel J, De Reuck J, Caemaert J. Lateralized effects of subthalamic nucleus stimulation on different aspects of speech in Parkinson's disease. Brain Lang 2003; 87: 253-8.

28. Skodda S, Schlegel U. Speech rate and rhythm in Parkinson's disease. Mov Disord 2008; 23: $985-92$.

29. Sofuwa O, Nieuwboer A, Desloovere K, Willems AM, Chavret F, Jonkers I. Quantitative gait analysis in Parkinson's disease: comparison with a healthy control group. Arch Phys Med Rehabil 2005; 86: 1007-13.

30. Stolze H, Klebe S, Poepping M, Lorenz D, Herzog J, Hamel W, Schrader B, Raethjen J, Wenzelburger R, Mehdorn HM, Deuschl G, Krack P. Effects of bilateral subthalamic nucleus stimulation on parkinsonian gait.Neurology. 2001; 10;57(1):144-6.

31. Wang E, Verhagen Metman L, Bakay R, Arzbaecher J, Bernard B. The effect of unilateral electrostimulation of the subthalamic nucleus on respiratory/phonatory subsystems of speech production in Parkinson's disease--a preliminary report. Clin Linguist Phon 2003; 17: 283-9.

32. Wolfe VI, Garvin JS, Bacon M, Waldrop W. Speech changes in Parkinson's disease during treatment with L-dopa. J Commun Disord 1975; 8: 271-9. 
Walking Velocity

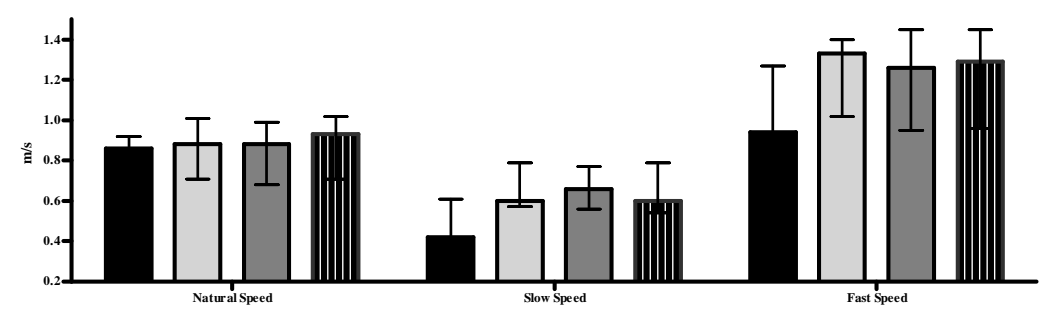

Step Length

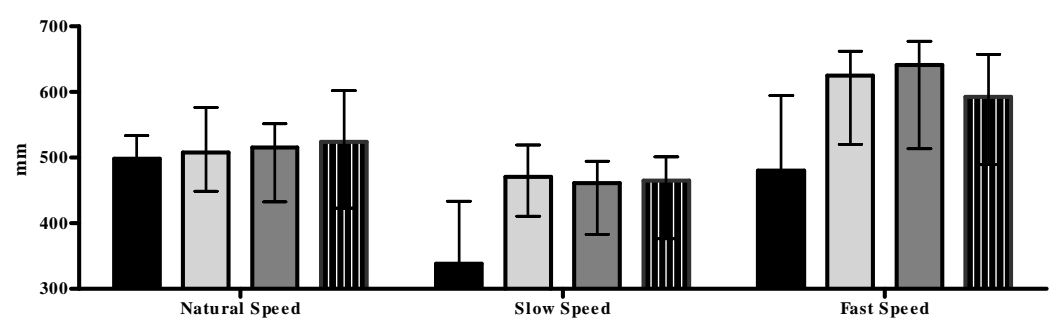

Walking Candence

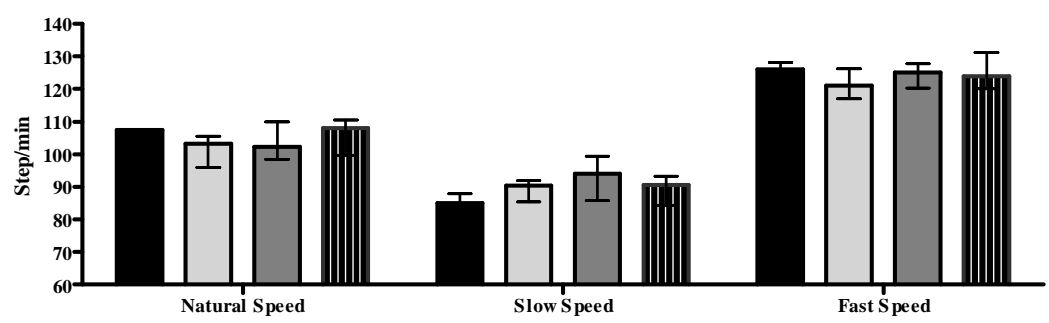

Figure 1 

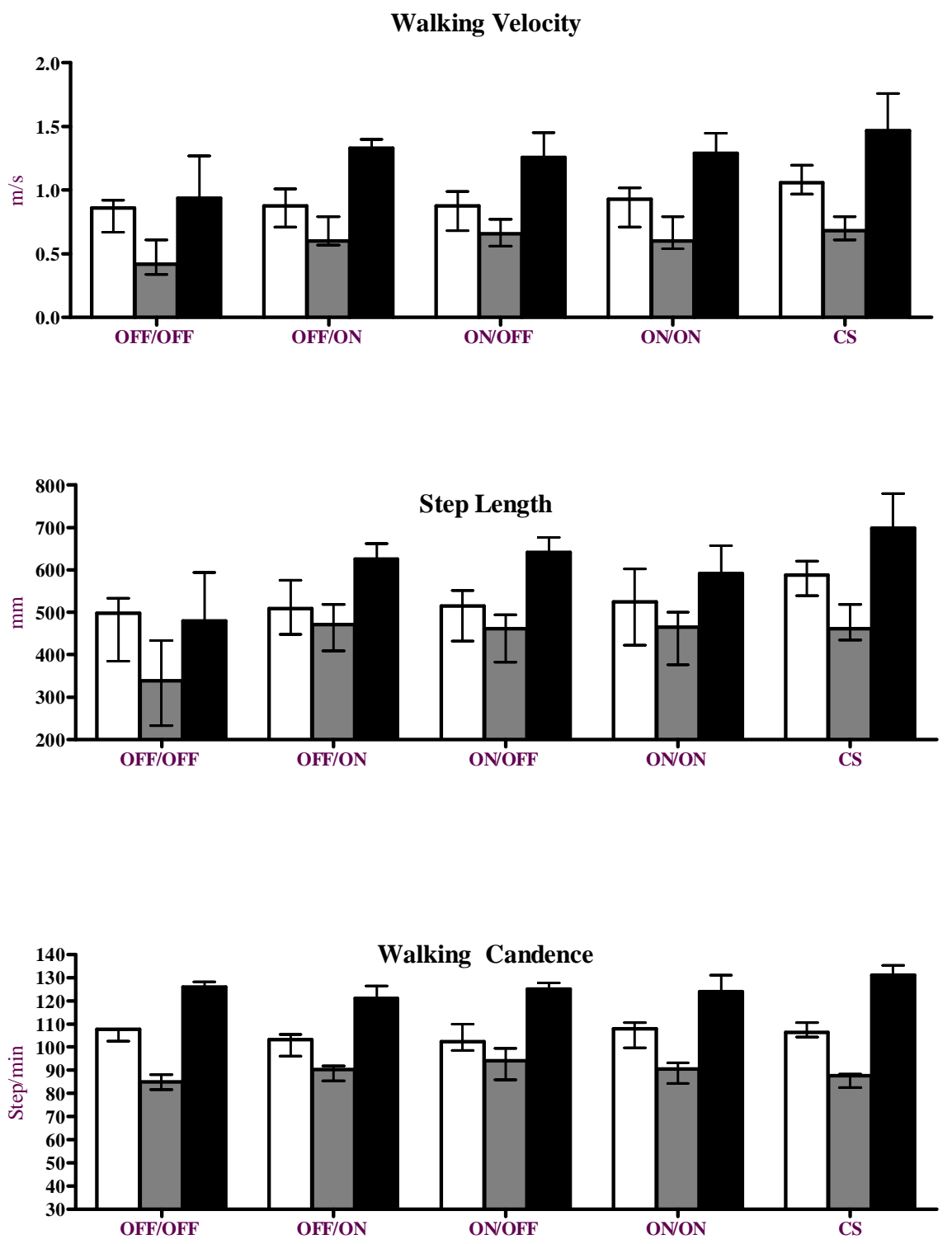

Figure 2 
Speech Velocity

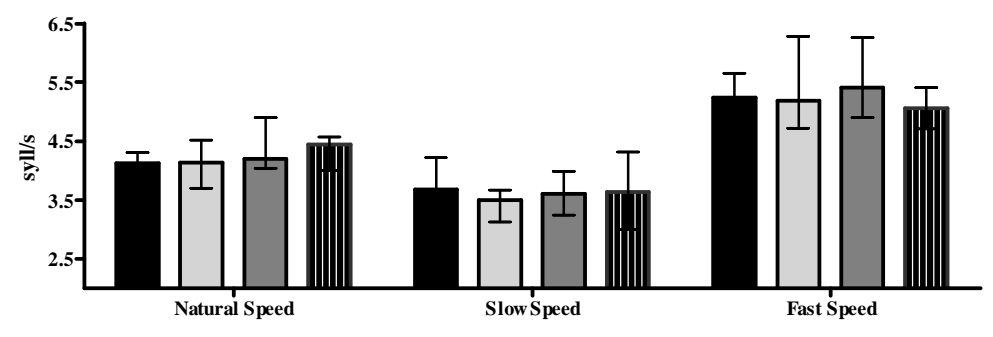

Interpause Speech Duration

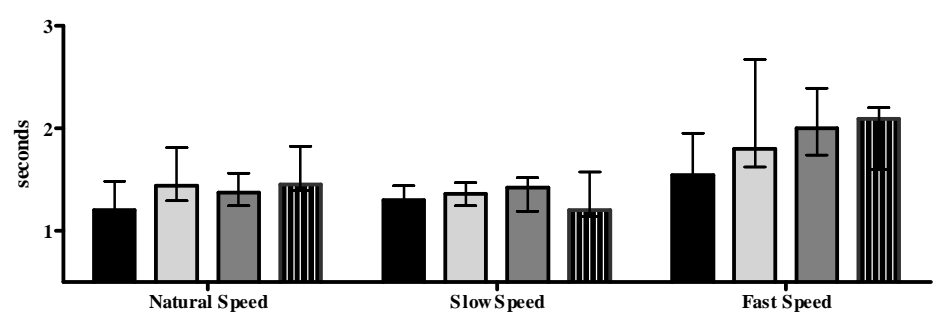

SPIR

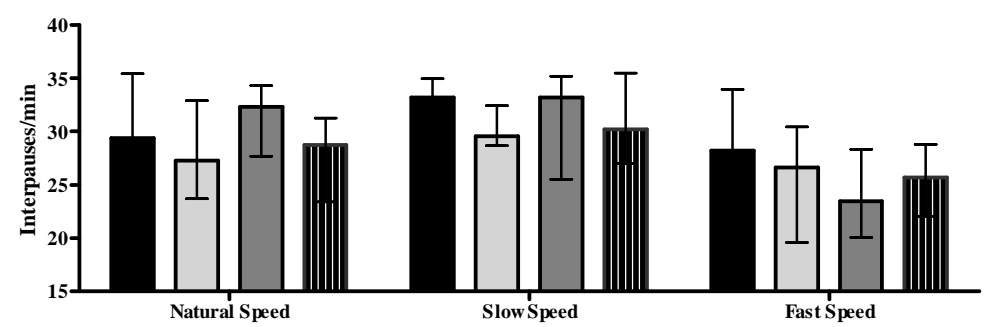

Figure 3 


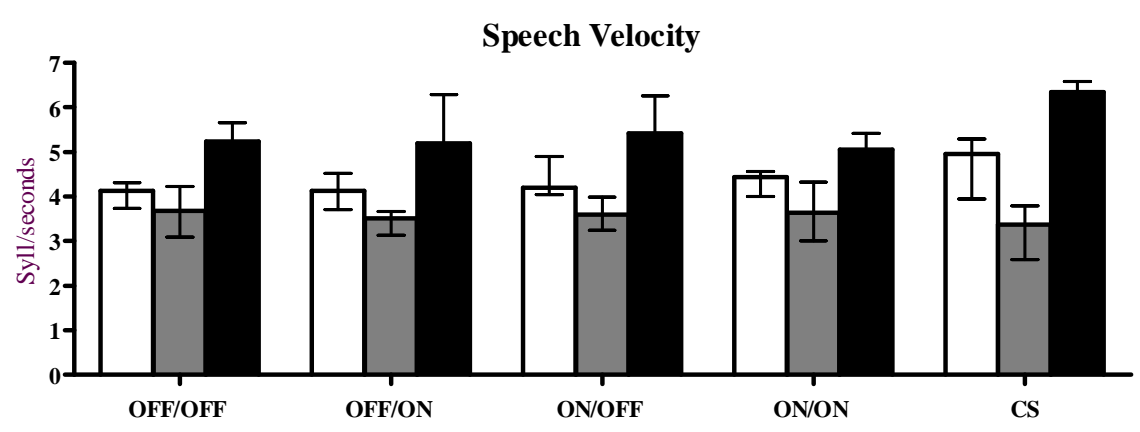

Interpause Speech Duration
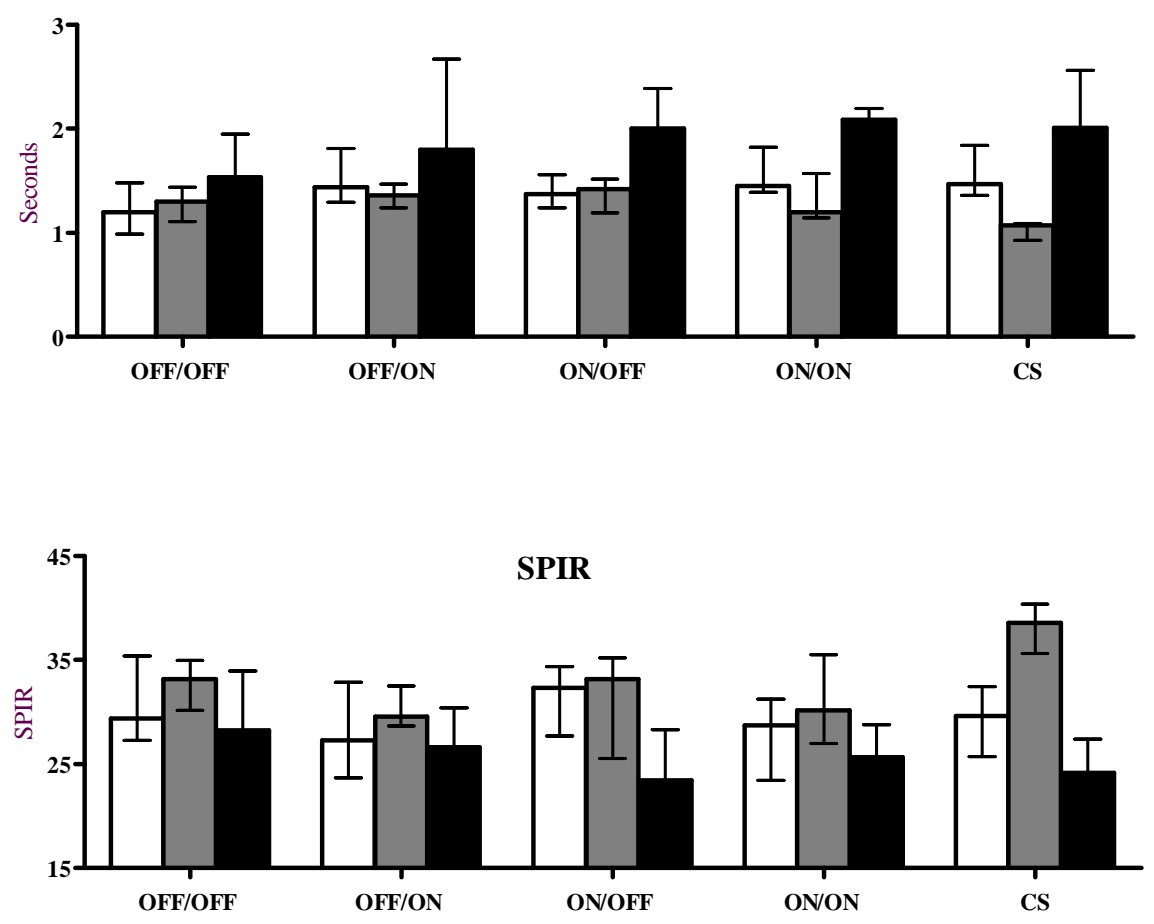

Figure 4 

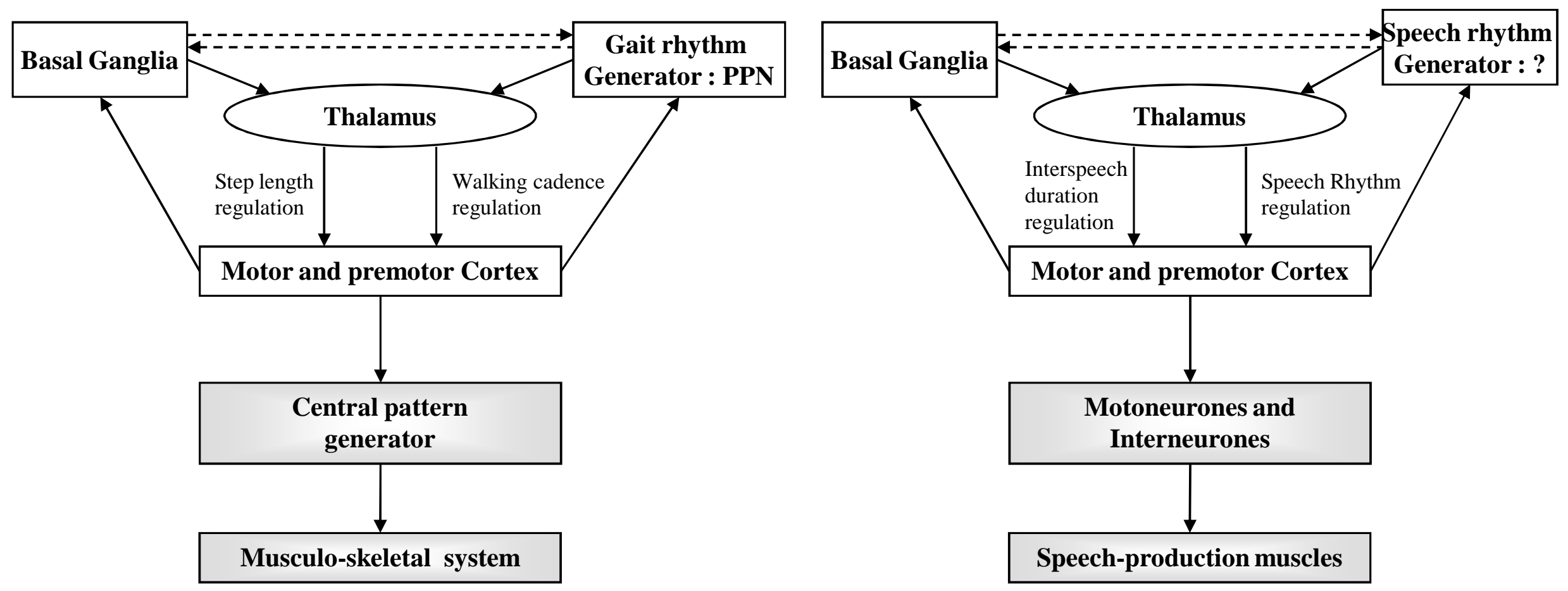

\section{Figure 5}

